\title{
Comments on Galileons
}

\author{
David Fairlie* \\ Department of Mathematical Sciences, \\ Science Laboratories, \\ University of Durham, \\ Durham, DH1 3LE, England
}

May 5, 2022

\begin{abstract}
The recent progress in the study of Galileons, i.e. equations of second order with an action invariant under a Galilean transformation is related to work on 'Universal Field Equations' 13] which are second order equations arising by an iterative procedure from arbitrary Lagrangians of weight one in their first derivatives. It is pointed out that the Galileon is simply a Kaluza-Klein reduction of a Universal Field Equation. An implicit solution to the equation of motion is presented, and a class of explicit solutions pointed out. The multi-field extensions of both types of equations are derived from a first order formalism, which is simply the substantive derivative of fluid dynamics.
\end{abstract}

\section{Introduction}

Recently there has been a spate of articles on the subject of Galileons, i.e. equations, second order in derivatives of a field $\pi$ with an action invariant under the infinitesimal transformation

$$
\pi(x) \mapsto \pi(x)+c+v_{\mu} x^{\mu}+\pi(x) v^{\mu} \partial_{\mu} \pi(x)
$$

with $x^{\mu}$ space-time co-ordinates, $v_{\mu}$ a constant vector and $c$ a constant. [1] [2] [3. In the literature two versions of the Lagrangian in $3+1$ dimensions are proposed; one starting with the Lagrangian

$$
\mathcal{L}_{2}=-\sqrt{1+(\partial \pi)^{2}}
$$

which is invariant up to a divergence under the transformation (11) and continuing with a heierarchy of increasingly complicated Lagrangians [2], ending

*e-mail: david.fairlie@durham.ac.uk 
with

$\mathcal{L}_{5}=-\gamma^{2}\left([\Pi]^{3}+2\left[\left[\Pi^{3}\right]-3[\Pi][\Pi]^{2}\right)-\frac{3}{2} \gamma^{4}\left(4[\Pi]\left[\pi^{4}\right]-4\left[\pi^{5}\right]-2\left([\Pi]^{2}-\left[\Pi^{2}\right]\right)\left[\pi^{3}\right]\right)\right.$

See also 4].The other starts with the Klein Gordon Lagrangian [5]. Here the notation $\left.\Pi_{\mu \nu}=\partial_{\mu} \partial_{\nu} \pi ;[\Pi]^{n}\right]=\operatorname{trace}\left(\Pi^{n}\right)$ and $\left[\pi^{n}\right]=\partial \pi . \Pi^{n-2} . \partial \pi$; i.e $\left[\pi^{3}\right]=$ $\partial_{\mu} \pi \partial^{\mu} \partial^{\nu} \pi \partial_{\nu} \pi$. The factor $\gamma$ is the inverse of $\sqrt{1+(\partial \pi)^{2}}$. The resulting equation of motion is $\mathcal{E}_{5}=0$ where

$$
\mathcal{E}_{5}=\gamma^{6}\left([\Pi]^{4}-6[\Pi]^{2}\left[\Pi^{2}\right]+8[\Pi]\left[\Pi^{3}\right]+3\left[\Pi^{2}\right]^{2}-6\left[\Pi^{4}\right]\right) .
$$

We shall discuss this procedure in the light of previous work on iterated Lagrangians, and relate it to these recent studies. This equation of motion can be very much more simply expressed thanks to a result on determinants, which is published in Lowell Brown's book, 6] but was independently found by myself; here illustrated for $4 \mathrm{x} 4$ matrices. Consider the determinant

$$
\operatorname{det}\left|\begin{array}{ccccc}
x^{4} & x^{3} & x^{2} & x & 1 \\
T_{4} & T_{3} & T_{2} & T_{1} & 4 \\
T_{3} & T_{2} & T_{1} & 3 & 0 \\
T_{2} & T_{1} & 2 & 0 & 0 \\
T_{1} & 1 & 0 & 0 & 0
\end{array}\right|
$$

where $T_{n}=\operatorname{trace}\left(T^{n}\right)$, and $T$ is a matrix of dimension $4 \times 4$. The expansion of this determinant is just 4 ! times the characteristic polynomial of the matrix $T$, and the coefficient independent of $x$ is just the determinant of $T$.

$$
\operatorname{det}(T)=-6 * T_{4}+8 * T_{3} * T_{1}+3 * T_{2}^{2}-6 * T_{2} * T_{1}^{2}+T_{1}^{4}
$$

But, if $T_{\mu \nu}=\frac{\partial^{2}}{\partial_{\mu} \partial_{\nu}} \pi=\Pi_{\mu \nu}$, this is just the operative factor in $\mathcal{E}_{5}$, so the equation of motion simply obtained by setting the Hessian $\operatorname{det}\left|\Pi_{\mu \nu}\right|=0$. This is also called the homogeneous Monge-Ampere equation and a general solution has been given by A.N. Leznov and myself 9 . I recently recognised an equivalent, and perhaps more elegant solution in a book of T.H. Chaundy, quite some time ago [10.

\subsection{Chaundy's solution}

The principle is illustrated by the $3 \times 3$ case. To solve

$$
\operatorname{det}\left|\begin{array}{lll}
\phi_{x x} & \phi_{x y} & \phi_{x z} \\
\phi_{x y} & \phi_{y y} & \phi_{y z} \\
\phi_{x z} & \phi_{y z} & \phi_{z z}
\end{array}\right|=0
$$

choose four arbitrary functions $f(u, v), g(u, v), h(u, v), k(u, v)$ constrained by the three relations

$$
\begin{aligned}
& x f(u, v)+y g(u, v)+z h(u, v)+k(u, v)=\phi(x, y, z) \\
& x f(u, v)_{u}+y g_{u}(u, v)+z h_{u}(u, v)+k_{u}(u, v)=0 \\
& x f_{v}(u, v)+y g_{v}(u, v)+z h_{v}(u, v)+k_{v}(u, v)=0 .
\end{aligned}
$$


Here subscripts denote partial differentiation with respect to $u, v$. Then the implicit solution of these equations for $\phi(x, y, z)$ is a solution to (6). This is easy to see; the three equations imply

$$
f(u, v)=\frac{\partial \phi(x, y, z)}{\partial x} ; \quad g(u, v)=\frac{\partial \phi(x, y, z)}{\partial y} ; \quad h(u, v)=\frac{\partial \phi(x, y, z)}{\partial z} .
$$

Since the right hand sides are three functions of two variables, there must be a relationship amongst them; i.e. there is a functional relationship among all the first derivatives of $\phi$, say $F\left(\phi_{x}, \phi_{y}, \phi_{z}\right)$. As we know this means that $\phi$ satisfies the Monge-Ampère equation. This is easily seen from the assumption of a relationship of the form

$$
F\left(\phi_{x}, \phi_{y}, \phi_{z}\right)=0
$$

Differentiation with respect to the variables $x, y, z$ respectively gives the three relations

$$
\begin{aligned}
& \frac{\partial F}{\partial \phi_{x}} \phi_{x x}+\frac{\partial F}{\partial \phi_{y}} \phi_{y x}+\frac{\partial F}{\partial \phi_{z}} \phi_{z x}=0 \\
& \frac{\partial F}{\partial \phi_{x}} \phi_{x y}+\frac{\partial F}{\partial \phi_{y}} \phi_{y y}+\frac{\partial F}{\partial \phi_{z}} \phi_{z y}=0 \\
& \frac{\partial F}{\partial \phi_{x}} \phi_{x z}+\frac{\partial F}{\partial \phi_{y}} \phi_{y z}+\frac{\partial F}{\partial \phi_{x}} \phi_{z z}=0 .
\end{aligned}
$$

The eliminant of these equations is simply the Monge-Ampere equation (6). We can also verify directly this by constructing the determinant of the matrix of second derivatives

$$
\operatorname{det}\left|\begin{array}{lll}
f_{u} u_{x}+f_{v} v_{x} & f_{u} u_{y}+f_{v} v_{y} & f_{u} u_{z}+f_{v} v_{z} \\
g_{u} u_{x}+g_{v} v_{x} & g_{u} u_{y}+g_{v} v_{y} & g_{u} u_{z}+g_{v} v_{z} \\
h_{u} u_{x}+h_{v} v_{x} & h_{u} u_{y}+h_{v} v_{y} & h_{u} u_{z}+h_{v} v_{z}
\end{array}\right|
$$

Evaluation of this determinant gives zero. It is also easy to see, by a similar technique that any homogeneous function of weight one, i.e which satisfies

$$
x \frac{\partial \phi}{\partial x}+y \frac{\partial \phi}{\partial y}+z \frac{\partial \phi}{\partial z}=\phi
$$

will also satisfy the equation (6) by a similar technique of differentiation by $x, y, z$ in turn. Interestingly, it is easy to see in this case that the Galilean transformation (11), not now necessarily infinitesimal also satisfies the equation (14) as, apart from the irrelevant constant, the additional terms are also of weight one, if $\pi$ is. One might worry as to whether the equality of the two different ways of evaluating mixed second derivatives introduces further constraints; for example

$$
\begin{aligned}
\frac{\partial^{2} \phi}{\partial x, \partial y} & =\frac{\partial f(u, v)}{\partial u} \frac{\partial u}{\partial y}+\frac{\partial f(u, v)}{\partial v} \frac{\partial v}{\partial y} \\
\frac{\partial^{2} \phi}{\partial y, \partial x} & =\frac{\partial g(u, v)}{\partial u} \frac{\partial u}{\partial x}+\frac{\partial g(u, v)}{\partial v} \frac{\partial v}{\partial x} .
\end{aligned}
$$


It turns out that the equality of the two expressions is automatically satisfied, and goes back to the equality of the mixed derivatives of $f, g, \ldots$ etc. Putting it more simply $f_{u} u_{y}+f_{v} v_{y}=g_{u} u_{x}+g_{v} v_{x}$. This is then a solution to the 3-dimensional Galileon equation

$$
\operatorname{det}\left|\frac{\partial^{2} \phi}{\partial_{\mu} \partial_{\nu}}\right|=0
$$

since this is just 6 times the determinant (14) in the case where $\mu, \nu$ run from $1 \ldots 3$. If the range is $1 \ldots n$, the determinant decomposes into the sum of $\frac{n !}{(n-3) !}$ Hessian determinants, and the same form of solution will hold by the elimination of $u, v$ from the equations

$$
\begin{aligned}
\phi\left(x_{\mu}\right)= & \sum_{\mu=1 \ldots n+1} x_{\mu} f^{\mu}(u, v) \\
& \sum_{\mu=1 \ldots n+1} x_{\mu} \partial_{u} f^{\mu}(u, v)=0 \\
& \sum_{\mu=1 \ldots n+1} x_{\mu} \partial_{v} f^{\mu}(u, v) .=0 .
\end{aligned}
$$

The construction in general can be easily inferred, and the analogous treatment of the solution of a related equation, described in the following section has been already described [12].

\section{Iteration of Lagrangians}

This version of a Galileon equation is actually simply a Kaluza Klein reduction of what J.Govaerts, A. Morozov and I called a 'Universal Field Equation' 13. We called it this because, starting with any Lagrangian dependent only upon $\pi$ and its first derivatives, and homogeneous of weight one in these derivatives, i.e.

$$
\sum \frac{\partial \mathcal{L}}{\partial\left(\partial_{\mu} \pi\right)} \partial_{\mu} \pi=\mathcal{L}
$$

subject to an iterative procedure, gives a UFE which is independent of the form of the initial Lagrangian. This obtained by iterating the Euler operator $\mathcal{E}$ acting on the Lagrangian

$$
\mathcal{E}=-\frac{\partial}{\partial \pi}+\partial_{i} \frac{\partial}{\partial \pi_{i}}-\partial_{i} \partial_{j} \frac{\partial}{\partial \pi_{i j}} \ldots
$$

(In principle the expansion continues indefinitely but it is sufficient for our purposes to terminate at the stage of second derivatives $\phi_{i j}$, since it turns out that the iterations do not introduce any derivatives higher than the second). We may start with a Lagrangian say, $\mathcal{L}=\sqrt{\sum_{\mu=1 \ldots 5}\left(\partial_{\mu} \pi\right)^{2}}$. Then the 5 fold iteration

$$
\mathcal{E} \mathcal{L} \mathcal{E} \mathcal{L} \mathcal{E}, \cdots, \mathcal{E} \mathcal{L}
$$


where each Euler operator acts on everything to the right yields the Universal Field Equation

$$
\operatorname{det}\left|\begin{array}{cccccc}
0 & \pi_{x_{1}} & \pi_{x_{2}} & \pi_{x_{3}} & \pi_{x_{4}} & \pi_{x_{5}} \\
\pi_{x_{1}} & \pi_{x_{1} x_{1}} & \pi_{x_{1} x_{2}} & \pi_{x_{1} x_{3}} & \pi_{x_{1} x_{4}} & \pi_{x_{1} x_{5}} \\
\pi_{x_{2}} & \pi_{x_{2} x_{1}} & \pi_{x_{2} x_{2}} & \pi_{x_{2} x_{3}} & \pi_{x_{2} x_{4}} & \pi_{x_{2} x_{5}} \\
\pi_{x_{3}} & \pi_{x_{3} x_{1}} & \pi_{x_{3} x_{2}} & \pi_{x_{3} x_{3}} & \pi_{x_{3} x_{4}} & \pi_{x_{3} x_{5}} \\
\pi_{x_{4}} & \pi_{x_{4} x_{1}} & \pi_{x_{4} x_{2}} & \pi_{x_{4} x_{3}} & \pi_{x_{4} x_{4}} & \pi_{x_{4} x_{5}} \\
\pi_{x_{5}} & \pi_{x_{5} x_{1}} & \pi_{x_{5} x_{2}} & \pi_{x_{5} x_{3}} & \pi_{x_{5} x_{4}} & \pi_{x_{5} x_{5}}
\end{array}\right|=0 .
$$

This equation possesses some interesting invariance properties; First of all it is signature blind; being the same in a Euclidean space-as a Lorentzian space-time. Also, any function of a solution is also a solution! This may be verified directly, but it arises from the solution procedure explained in [12. Now in a Kaluza Klein reduction we may choose a gauge in which $\frac{\partial \pi}{\partial x_{5}}=1$. In this case the last row and column of (23) have only two nonzero elements in the $(1,6)$ and $(6,1)$ positions, and these are unity. The resulting determinant simply reproduces (6), the Hessian.

\section{Connection with earlier results}

These results are easily understood as examples of the main results of [13] quoted here; 'In two recent papers [13, 15, the first with A. Morozov, hierarchies of

Lagrangian field theories with the following properties were introduced.

1. i) In any of these hierarchies, the Lagrangian at any given level - except of course at the first level - is essentially proportional to the equations of motion of the Lagrangian at the previous level (hence the name Euler hierarchies).

2. ii) The proportionality factor mentioned in i) is essentially the very first Lagrangian in the hierarchy.

3. iii) In any of these hierarchies, Lagrangians depend on fields only through their first and second derivatives, but not on derivatives of higher order nor on the fields themselves. The first Lagrangian only depends on first derivatives of the fields. The dependence of each of the other Lagrangians on second derivatives is multilinear, and of order equal to the number of times an equation of motion has been taken to reach that level in the hierarchy.

4. iv) All these hierarchies are finite, i.e. the iterative procedure implied by i) iii) terminates after a finite number of steps.

5. v) For each hierarchy, the last non trivial equations of motion are universal, namely, up to a factor, they are independent of the initial Lagrangian out of which the hierarchy is constructed. The associated infinite number of conserved charges - corresponding to the freedom in the choice of initial Lagrangian - suggests the possible integrability of these universal equations (equations of motion are indeed always current conservation equations for Lagrangians without an explicit dependence on fields). 
Specifically, hierarchies with these properties were shown to exist in the following cases:

- a single field $\phi$ in $d$ dimensions, with an arbitrary initial Lagrangian (function of first derivatives only) [13,

- a single field $\phi$ in $(d+1)$ dimensions, the initial Lagrangian now being an arbitrary homogeneous weight one function of its arguments [13]

- $(d+1)$ fields $\phi^{a}$ in $d$ dimensions, with an arbitrary reparametrisation invariant initial Lagrangian [15].

The hierarchies associated with these three cases terminate after $d$ steps, with the following universal equations:

1.

$$
\operatorname{det} \phi_{i j}=0
$$

2.

$$
\operatorname{det}\left(\begin{array}{cc}
0 & \phi_{j} \\
\phi_{i} & \phi_{i j}
\end{array}\right)=0
$$

3.

$$
\operatorname{det}\left(J_{a} \phi_{i j}^{a}\right)=0 .
$$

Here, $\phi_{i}$ and $\phi_{i j}$ denote the partial derivatives $\left(\partial \phi / \partial x_{i}\right),\left(\partial^{2} \phi /\left(\partial x_{i} \partial x_{j}\right)\right)$ of the field $\phi$ with respect to the $d$ or $(d+1)$ coordinates $x_{i}$. (The same applies of course to the fields $\phi^{a}$, and obviously the indices $i$ and $j$ in the equations above refer to lines and columns respectively of the corresponding matrices. The usual summation convention over repeated indices is assumed throughout). In (26), the quantities $J_{a}$ are the Jacobians

$$
J_{a}=(-1)^{d} \epsilon_{a b_{1} b_{2} \cdots b_{d}} \phi_{1}^{b_{1}} \phi_{2}^{b_{2}} \ldots \phi_{d}^{b_{d}} .^{\prime}
$$

Equation (25) is a generalisation of the original two dimensional Bateman equation [13] (corresponding to (25) with $(i=1,2)$ ) which is also known to be integrable [13. Finally, equation (26) is a generalisation to a $(d-1)$-dimensional membrane in a $(d+1)$-dimensional spacetime of the (universal) equation of motion for a parametrised particle in a flat two dimensional spacetime (corresponding to $d=1$ ), the latter clearly being also integrable. Note that (26) includes a universal equation for a string theory in three dimensions $(d=2)$, and a universal equation for a membrane theory in four dimensions $(d=3)$.

Remarkably, the three classes of universal equations above are invariant under arbitrary linear $G L(n)$ transformations in the variables $x_{i}$ as well as in the fields $\phi^{a}$, even though neither the initial nor the successive Lagrangians in the corresponding hierarchies would generally possess these symmetries. The equations above thus provide examples of equations of motion admitting an infinite number of Lagrangians, with symmetry properties that these Lagrangians need not possess. 


\subsection{Other starting points}

In a second paper Hinterbickler et al [5, the authors start from a simple massless Lagrangian, $\mathcal{L}_{2}=\left[\pi^{2}\right]$ in 4 dimensional space-time and generate a sequence of Lagrangians with second order equations of mmotion. These actually follow from the iterative procedure described above; and are obtained by setting each $\mathcal{E}_{j}=0$, where

$$
\begin{aligned}
\mathcal{E}_{2} & =-2[\Pi] \\
\mathcal{E}_{3} & =-3\left([\Pi]^{2}-\left[\Pi^{2}\right]\right) \\
\mathcal{E}_{4} & =-2\left([\Pi]^{3}-\left[\Pi^{3}\right]-3[\Pi][\Pi]^{2}\right) \\
\mathcal{E}_{5} & =-\frac{5}{6}\left([\Pi]^{4}-6\left[\Pi^{4}\right]-8[\Pi][\Pi]^{3}+3\left[\Pi^{2}\right]^{2}\right) .
\end{aligned}
$$

The last of these, $\mathcal{E}_{5}=0$, is identical, up to an irrelevant factor to equation (4) !.The other equations are simply given (again up to an irrelevant numerical factor) by

$$
\mathcal{E}_{n+1}=\operatorname{det}_{n}\left|\frac{\partial^{2} \pi}{\partial x_{\mu} \partial x_{\nu}}\right|
$$

where the matrix involved is an $n \times n$ matrix. In the above example $\mathcal{E}_{5}$ is just 4 ! times the Hessian; in the previous case, $\mathcal{E}_{4}$ is just 6 times the sum of the four $3 \times 3$ Hessians formed by the second derivatives with respect to the four possible subsets of three of the variables $x_{1}, x_{2}, x_{3}, x_{4}$. Likewise $\mathcal{E}_{3}$ is just

$$
\mathcal{E}_{3}=3\left(\sum_{\mu=1 . .4} \sum_{j=1 . .4}\left(\partial_{\mu \mu} \pi \partial_{\nu \nu} \pi-\partial_{\mu}^{\nu} \pi \partial_{\nu}^{\mu} \pi\right)\right)
$$

These terms are just proportional to the coefficients in the expansion of the characteristic polynomial of the matrix (31). These equations are solved by a similar technique to that above for $\mathcal{E}_{5}=0$. These sequences of Lagrangians and equations of motion are effectively the same as those of [4. These results are easily understood as examples of the main results of [14] as is the fact that the equations of motion starting with a square root Lagrangian are of similar form to those starting from the Klein Gordon Lagrangian. (see [16]).

\subsection{Multi-Galileons,}

The theory has already been extended to the case of many fields $\pi^{i}[3]$ [ [5] From the point of view presented here, just as the single-field case was a KaluzaKlein reduction of the Universal Field Equation, so are the multi-Galileon fields a reduction of the multi-field UFE, presented in [15, by setting the derivatives of the fields in the $x_{5}$ th direction to unity. The multi-field UFE permits a first order formalism; The set of equations of hydrodynamic type;

$$
\frac{\partial u^{i}}{\partial x_{0}}+\sum u^{j} \frac{\partial u^{i}}{\partial x_{j}}=0
$$


are just the equations of conservation of momentum in an inviscid, incompressible fluid. Differentiation with respect to $x_{j},(j=0 \ldots n)$ gives a set of second order differential equations, and the eliminants of those together with (32) are the multi-field equations. The equations (32) admit an easy implicit solution. The details may be found in [11. Indeed, multi-field UFE, with up to $n$ fields, $k$, say, may be obtained from the equations (32) by setting $u^{j}, j=k+1 \ldots n$ as functions of $u^{i}, i \ldots k$, and forming the eliminants of these equations together with their derivatives with respect to the independent variables. In particular the single field UFI comes from the equation

$$
\frac{\partial u}{\partial x_{0}}+\frac{\partial u}{\partial x_{1}}+\sum_{j=2 \ldots n} u^{j}(u) \frac{\partial u}{\partial x_{j}}=0
$$

Differentiation with respect to $x_{k}, k=0 \ldots n$ yields the equations

$$
\frac{\partial^{2} u}{\partial x_{0}, \partial x_{k}}+\frac{\partial^{2} u}{\partial x_{1} \partial x_{k}}+\sum_{j=2 \ldots n} u^{j}(u) \frac{\partial^{2} u}{\partial x_{j} \partial x_{k}}+\frac{\partial u}{\partial x_{k}}\left(\sum_{j=1 \ldots n} \frac{\partial}{\partial u} u^{j}(u) \frac{\partial u}{\partial x_{j}}\right)=0
$$

Elimination of the coefficients $1, u^{j}(u), \sum_{j=1 \ldots n} \frac{\partial}{\partial u} u^{m}(u) \frac{\partial u}{\partial x_{m}}$ yields the UFI

$$
\operatorname{det}\left|\begin{array}{cc}
0 & \frac{\partial u}{\partial x_{i}} \\
\frac{\partial u}{\partial x_{j}} & \frac{\partial^{2} u}{\partial x_{i} \partial x_{j}}
\end{array}\right|=0
$$

The Galileon can be obtained by a similar process of elimination, starting from (34). Making the assumption $u^{n}=1$, and also imposing the side condition

$$
\sum_{m=1 \ldots n-1} \frac{\partial u^{m}(u)}{\partial u} \frac{\partial u}{\partial x_{m}}+\frac{\partial u^{n}(u)}{\partial u}=0
$$

the last equation of (34) vanishes and the eliminant is the determinant of the $n \times n$ matrix $\frac{\partial^{2} u}{\partial i \partial j}$ to zero. Thus the Galileon also has roots in fluid dynamics.

\section{Conclusions}

The Galileon equations can be viewed as arising from a Kaluza Klein reduction of the universal field equation, (or more prosaically, as Chaundy has called it [10, the bordered determinant) by setting the derivative of the fields with respect to the fifth co-ordinate to unity. This universal field equation admits a first order formalism in terms of an equation of hydrodynamic type, and is solved implicitly. The single field Galileon equation is a sum of Hessians set to zero and also admits implicit solutions. The universal field equation arises in the theory of developable surfaces, and has also connections with Dirac-BornInfeld theory 17] 18. Very recently a paper has appeared [19] showing that the Galileon actions and their covariant generalisations can also be obtained as a Kaluza Klein compactification of higher dimensional Lovelock Gravity. 


\section{Acknowledgment}

I should like to acknowledge Jan Govaerts for useful comments.

\section{References}

[1] A. Nicolis, R. Rattazzi, and E. Trincherini, Phys. Rev. D79, 064036 (2009), ArXiv:0811.2197[hep-th].

[2] C. de Rham and A.J.Tolley, DBI and Galileon reunited, JCAP 1005: 015, arXiV:1003.5917[hep-th] (2010).

[3] C. Deffayet, S. Deser, G. Esposito-Farese Arbitray p-form Galileons Phys. Rev. D 82061501 (R) arXiv:1007.5278 [gr-qc](2010)

[4] G.L.Goon,K Hinterbichker and Mark Trodden, Stability and superliminality of spherical DBI galileon solutionsarXiv:1008.4580v1[hep-th])

[5] K.Hinterbickler, M.Trodden and D.Wesley Multi-field galileons and higher co-dimension branes, arXiv:1008.1305v1 [hep-th](2010)

[6] L.S. Brown, Quantum Field Theory, Cambridge University Press (1992)

[7] A. Padilla, P. M. Saffin and Shuang-Yong Zhou, Bi-Galileon Theory I:motivation and formulation JHEP 1012:031, arXiV:1007.5424[hepth] (2010).

[8] A. Padilla, P. M. Saffin and Shuang-Yong Zhou, Multi-Galileons, solitons and Derrick's theorem arXiV:1008.0745[hep-th](2010).

[9] D.B. Fairlie and A.N. Leznov, General solutions of the Monge-Ampère equation in $n$-dimensional space, Journal of Geometry and Physics. 16 (1995) 385-390. hep-th/9403134

[10] T. Chaundy The Differential Calculus The Clarendon Press, Oxford (1935).

[11] D.B. Fairlie, A Universal Solution, J. Nonlin. Math. Phys9 (2002) 256-261.

[12] D.B. Fairlie, Implicit solutions to some Lorentz invariant nonlinear equations revisited, $J$ Nonlinear Math. Phys. 12 (2005) 449456 math-ph/0412005

[13] D.B. Fairlie and J. Govaerts and A. Morozov, Universal Field Equations with Covariant Solutions, Nuclear Physics B 373 (1992) 214-232.

[14] D.B. Fairlie and J. Govaerts, Euler Hierarchies and Universal Equations, Journal of Mathematical Physics.33 (1992) 3543-3566.

[15] D.B. Fairlie and J. Govaerts, Universal Field Equations with Reparametrisation Invariance,

Physics Letters B 281 (1992) 49-53. 
[16] D.B. Fairlie, Dirac-Born-Infeld Equations, Phys .Lett. B456, (1999) 141146

[17] L.M. Baker and D.B. Fairlie, Companion Equations for Branes, (1999) hep-th/9908157, Journal of Mathematical Physics 41 (2000) 4284-4292.

[18] L.M. Baker and D.B.Fairlie, Hamilton-Jacobi equations and Brane associated Lagrangians, Nuclear Physics B596 (2001) 348-364.

[19] K. Van Acoleyen and J. Van Doorsselaere, Galileons from Lovelock actions, arXiv:1102.0487v2 [gr-qc]. 